\title{
The load and capacity model of healthcare delivery: considerations for the crisis management of the COVID-19 pandemic
}

\author{
Joerg Steier ${ }^{1,2}$, John Moxham ${ }^{1}$ \\ ${ }^{1}$ CHAPS, Faculty of Life Sciences and Medicine, King's College London, UK; ${ }^{2}$ Lane Fox Unit/Sleep Disorders Centre, Guy's \& St Thomas' NHS \\ Foundation Trust, London, UK \\ Correspondence to: Joerg Steier, FRCP, MD, PhD. Lane Fox Unit, Ground Floor, South Wing, Guy's \& St Thomas' NHS Foundation Trust, \\ Westminster Bridge Road, London SE1 7EH, UK. Email: Joerg.steier@kcl.ac.uk. \\ Provenance and Peer Review: This article was commissioned by the editorial office, fournal of Thoracic Disease. Given the wide concern and pressing \\ importance of research relating to COVID-19, this editorial was managed via fast-track communication pathway and internal review.
}

Submitted May 12, 2020. Accepted for publication Jun 10, 2020.

doi: $10.21037 /$ jtd-2020-054

View this article at: http://dx.doi.org/10.21037/jtd-2020-054

\section{Introduction}

In the early phase of the year 2020, a novel virus outbreak led to a worldwide pandemic with millions of confirmed cases (1) that caused large proportions of the world population to be in temporary lockdown. With non-essential travel discouraged and everyone but key workers staying at home the world economy came to a sudden pause (2). The containment of the virus, a novel coronavirus named COVID-19, required quick resource re-allocation on a large scale and was prioritised on every level of healthcare delivery, first identified in East Asia. As the outbreak continued the epicentre shifted to Europe and the Middle East, and eventually affected the Americas (3). It led to restrictions on public life previously unimaginable during times of peace (4). Schools were closed, work from home was strongly encouraged, and non-essential travel was forbidden; some regions, and even countries, were entirely locked down for weeks or months (5).

Restrictions to public life were necessary to avoid many people being infected at the same time which would have led to a collapse of the healthcare system. With no immunity to this new virus in the general population and many people being infected and potentially requiring critical care treatment at the same time, it was essential to reduce the rate of new infections (6).

In this document, we discuss factors that must be considered during pandemic outbreaks, and introduce the load-to-capacity model for healthcare delivery, to explain how saturation of the system can be averted (7), and explore what the implications are for long-term investment decisions in healthcare.

\section{Load-to-capacity model}

The structure and function of national healthcare systems are important for effective healthcare delivery when facing a sudden and substantial surge in demand. The model (Figure 1) shows the resources that need to be considered when addressing the demand in a pandemic.

\section{Capacity}

In non-pandemic times, capacity in most countries is largely, but not exclusively, limited by the investment into healthcare as provided by a national or insurance-based system. In times of a pandemic, governments and healthcare policymakers are likely to adjust healthcare-related budgets to try and address the acute demand.

\section{Staff}

Medical staff with appropriate expertise and skillsets in sufficient numbers beyond the regular hospital and critical care capacity, is essential to cope with pandemic demand. Critical care skilled staff, often redeployed from other services, needs to be available if the most severe cases of infection are to be treated according to best standards. The workforce varies in developed countries by a factor of two for doctors, between 2.1-4.3 per 1,000 population, and by about the same factor for nurses, between $8.2-17.4$ per 


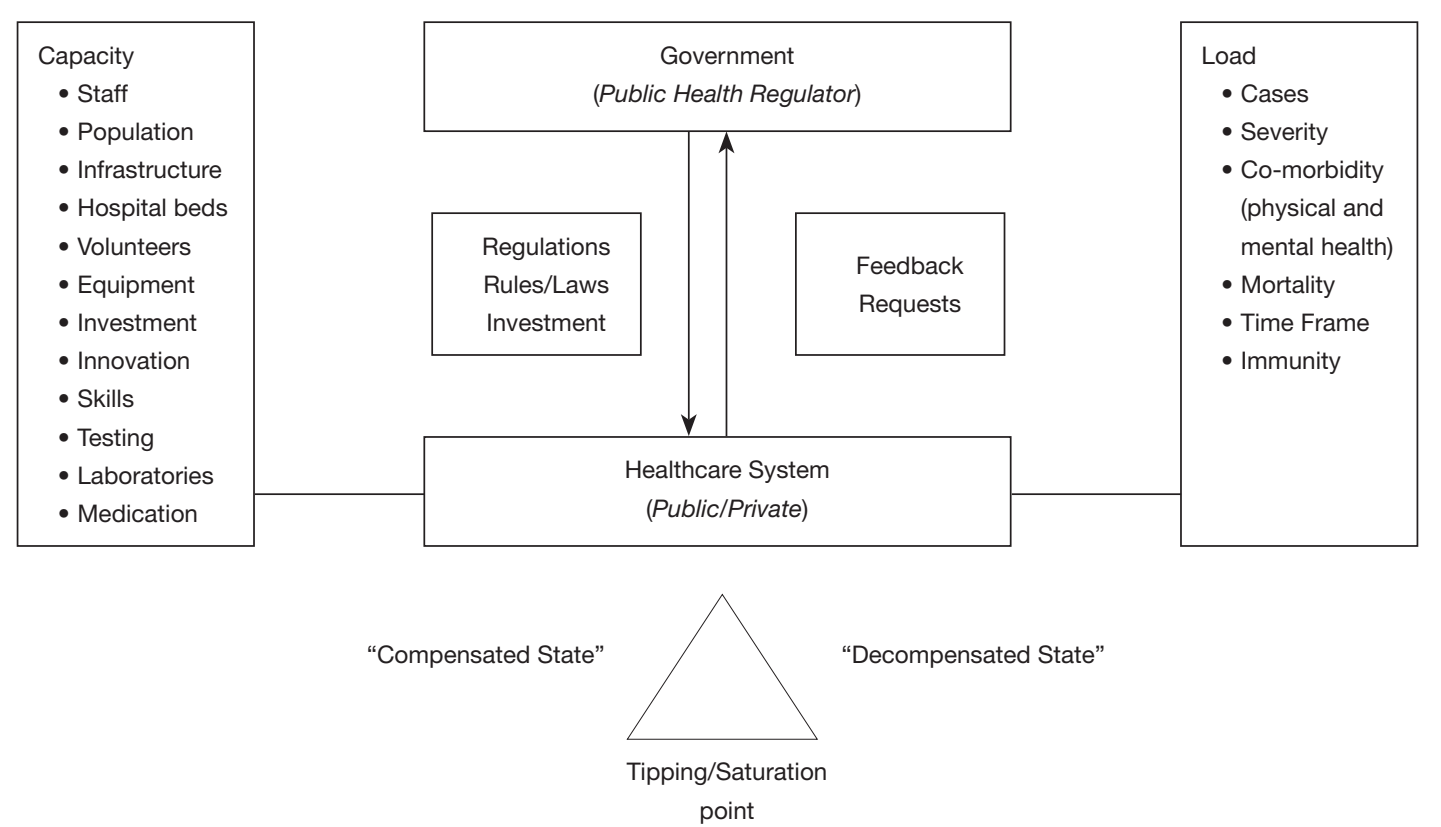

Figure 1 Load-to-Capacity model of healthcare systems. The system remains in a compensated or balanced state as long as the capacity can meet the load, but if the load increases sufficiently a tipping point is reached and healthcare delivery fails.

\section{1,000 population (8).}

In non-pandemic times, intensive care units are guided by established care pathways, specialist nurse and medical cover, close relationships with other operational delivery networks, interdependency and close proximity to colocated services (9). In a pandemic, most essential services, particularly critical care units, will be overstretched and expand to additional wards and clinical areas (e.g., in operation theatres) that will need to be served for by redeployed staff.

In such situations, the "level of care", defined by the ratio of critical care nurses per patient, typically defined as $1: 1$ for level III beds, 1:2 for level II beds and 1:3 for level I beds (10) will quickly be diluted. In the NHS Nightingale hospital in London, UK, a new hospital that was set up to cope with the COVID-19 crisis, the ratio of critical care nurse per patient was initially planned to be 1:6 in the worst case scenario (11).

The general public has an important part to play in supporting the capacity of the healthcare system, both by influencing resource allocation decisions and by volunteering (12), as well as by following government guidance. During exceptional circumstances, the military may also be engaged for logistical and operational support to strengthen capacity.

\section{Hospital and critical care bed capacity}

There are finite hospital beds in non-pandemic times. However, in pandemic times inflated bed capacity means that lives can be saved. Total bed capacity varies approximately by a factor 10 between all countries, from 1.4 to 13.1 per 1,000 people. There are sparse published data on critical care bed capacity, but wherever data are available the rate differs between 3.6 to 47.1 beds per 100,000 citizen, with the majority of countries ranging between 5 and 15 beds per 100,000 (13).

Bed capacity is key factor that determines whether patients can be looked after in hospitals during a pandemic. In case of clinical deterioration, it is particularly the critical care bed capacity that is essential to keep patients alive whilst their immune system clears the virus and vital organs, particularly the lungs, recover. However, hospital occupancy rates are to be about $85 \%$ in non-pandemic times (14). In order to provide best possible care for infected patients, it is therefore essential to inflate the critical care bed capacity quickly during a pandemic.

A limiting factor for critical care bed availability is the turnover time of affected cases. In patients with COVID-19 this is, in most cases, between 7-14 days. When critical care bed capacity is saturated the ceiling of care needs to be considered for any subsequent admissions, which requires 
triage and, unfortunately, impacts significantly on overall mortality. In the UK, the National Institute for Health and Care Excellence, NICE, published guidance that patients with a Clinical Frailty Score (CFS) of less than five points, which indicates that patients are not dependent, should be considered for critical care support, as it was likely to be beneficial. However, NICE also recommended that for patients with a score of five or more points on the CFS, which indicates mild frailty, "there is uncertainty regarding the likely benefit of critical care organ support" (15).

\section{Population}

The demographics of a specific population matter in the current pandemic. Age, gender, ethnicity, comorbidities, density and exposure to urban areas, physical and mental health as well as compliance with public health guidance define potentially vulnerable or at risk cohorts, but these factors may also indicate solutions for successful risk stratification and non-vaccination measures. It is important to prepare the population to avoid anxiety and unnecessary actions. This is best achieved by regular open communication to explain decisions taken, current developments in the dynamics of the pandemic, and guidance on strengthening mental and physical health during lockdowns, to keep people motivated, involved and active (16). It has to be considered that overall wellbeing includes financial and social aspects that are of great importance and will contribute to people's compliance with long-term restrictions to their lives. The full extent the impact of a pandemic lockdown, either individually due to loss of income and employment, or for the economy due to reduced trade and business activity, may only become apparent once the disease is controlled, but can have a hugely detrimental effect on non-infected parts of the population.

\section{Equipment and consumables}

Logistical requests of large scale orders during pandemic times can cause problems in affected countries and this affected particularly Personal Protective Equipment (PPE) and ventilators (17):

* In the most severe cases of COVID-19, intensive care ventilators are licensed to invasively ventilate intubated patients. Although there are other kinds of ventilators, such as non-invasive ventilators, continuous positive airway pressure (CPAP) machines and home mechanical ventilators, it is the ventilators that are licensed for critical care that are essential to keep very sick patients alive.

* In the initial stages of the pandemic there was not enough PPE available to protect frontline staff and key workers. Even at the peak the Royal College of Physicians in London, having surveyed NHS staff, found that only $78 \%$ had access to adequate PPE (18). Sensible professional guidance on appropriate PPE should not be influenced by policymakers who are facing limited supplies; this can cause confusion about what PPE pieces are required. A lack of PPE or inappropriate guidance on PPE exposes frontline staff to avoidable infections, and sometimes death, with many healthcare workers off sick or selfisolating. During the early phase of the pandemic, about $18-21 \%$ of NHS staff had to take time off work due to infection or self-isolation (18).

At the peak of the pandemic oxygen availability also required serious consideration, as maximal flow rates were reached in some hospitals. High-flow nasal oxygen, CPAP and non-invasive ventilation machines were similarly in high demand, but due to the aerosol generating nature of these therapies some hospitals had to put restrictions on where and how these devices could be used.

\section{Testing and contact tracing}

Testing for COVID-19 is essential to understand the prevalence of the disease, the affected areas and the hospitalization rates, and it is also required to accurately assess mortality. Some countries did not test many patients, particularly in the beginning of the pandemic. However, other countries recognized early that by testing and identifying cases, isolation measures were more efficient and case identification and tracing contacts could help to avoid the spread of the disease, slow and limit the outbreak.

Tests performed per 1,000 of the population differed significantly between countries; some countries performed 3-8 tests per 1,000 citizens during the initial period of the pandemic while other countries achieved between 13-18 tests per 1,000 citizens relatively early (19). Once affected countries agreed that testing was helpful and available daily test capacity then became the next issue. Existing facilities needed to adjust their laboratories to accommodate large scale requests. Despite limited accuracy testing can provide relative certainty with the diagnosis. It is another tool to try and contain the disease, allocate resources and effort, and it allows self-isolating healthcare staff to return safely to the frontline once cleared. Amongst respondents to a survey in the UK National Health Service, 
about $31 \%$ of the workforce had access to testing at the height of the pandemic (17). The ratio of positive tests compared to all tests taken is a good indication of whether sufficient case numbers are tested; a high rate of positive results would indicate that it is likely there are many more cases that go unnoticed and untested. It remains uncertain to what extent modern technology, e.g., smartphone applications, will be helpful in tracing contacts during a pandemic in the future, as civil rights and liberties need to be carefully balanced with the greater good of public health.

\section{Investment}

Strategic planning and investment in a modern healthcare system facilitates the retention of skilled medical and technical staff, allows the use of the latest equipment, the latest evidence-based tests and treatments prepare the system for a sudden surge of patients during a pandemic. Pre-existing infrastructure can be scaled quickly. However, annual investment into healthcare in western systems differs remarkably between countries, US\$3,377-US\$9,403 per capita (mean US\$5,419) (8).

New drugs become available faster if investment into research and development has successfully established an infrastructure that can respond to a sudden change in demand. Governance structures, ethical review boards and procedures to record side effects and respond to adverse events need to be already in place prior to a pandemic.

Once the acute phase of the infection is over, rehabilitation and weaning programmes for survivors of critical care, with focus on physical and mental health, are essential to restore quality of life (20). Post critical care rehabilitation requires time, space, physiotherapists, multidisciplinary guidance, as well as psychological counselling. Weaning units that specialize in ventilation and rehabilitation facilitate complex discharges and act as an appropriate interface between acute and community care.

Lastly, the placement of patients who require ongoing care or ventilation following discharge from hospital requires appropriate investment. In parallel to those affected directly by the pandemic infection, there is an additional need to support the general public, who have not had the infection, by putting in place furlough schemes, unemployment support and engaging banks to supply the public and businesses with uncomplicated credits to aid employment for those adversely affected by the impact of the public lockdown.

The capacity assessment of a healthcare system is critically important if it is to be prepared for a pandemic. Such assessments are important and necessary during non-pandemic times, as prevention is massively cost-effective compared with the impact of an unprepared system for a pandemic.

\section{Load}

The demand on any healthcare system varies with time, affected by the seasons (influenza) and disease states and by the demographics of the population. Treatment and care need to be cost-effective to sustain an equitable healthcare system. Cost-effectiveness can be assessed and calculated. In evidence-based medicine, healthcare structure and function is continuously assessed to ensure that healthcare provision remains affordable for the entire population. This is achieved by examining whether specific diagnostics/therapeutics can be rolled out across a nation. Individual countries typically set specific threshold levels for cost-effectiveness depending on their budgets, however, quality adjusted life years (QALYs) are an internationally accepted measure used to compare interventions and to judge whether treatments are affordable (21). During the COVID-19 pandemic, with sudden and unpredictable changes in the load on the healthcare system, new evidence needs to be considered on a daily base.

\section{Cases}

The case load on a healthcare system is the single most significant parameter. In a typical flu pandemic, the season is often stretched out over a period of several months (e.g., October to April) and infected patients do not present all at once. In the current pandemic with COVID-19, the entire population had no antibodies to the virus and the infection flared up in regions like a tsunami wave, affecting regions and then entire countries and their population within days and weeks.

The basic reproduction number, $\mathrm{R}_{0}$, is important, as it is influenced by the exponential increase in infected patients during the outbreak, factors such as the latent infectious state and the infection period. In COVID-19, $\mathrm{R}_{0}$ was initially described to be between 1.4 and 3.8 in Wuhan/ China, but during the outbreak in New York/US it was estimated to be 5.7 (95\% Confidence interval: 3.8-8.9) (22). $\mathrm{R}_{0}$ is also used to estimate the portion of the population that is required to have antibodies, either by prior infection or vaccination, to lead to disease extinction $\left(1-1 / \mathrm{R}_{0}\right)$; for $\mathrm{R}_{0}=5.7$ this proportion is $82 \%$ (22). To control an outbreak over time, $\mathrm{R}_{0}$ needs to be consistently below 1.0.

COVID-19 can resolve in many patients with minor symptoms, but in some cases it causes a sudden and severe 
acute respiratory distress syndrome (ARDS) like picture with organ failure that requires intubation and ventilation (23). In these cases, critical care bed and ventilator availability define the ceiling of care. In addition, renal replacement therapy and access to extra-corporal membrane oxygenation (ECMO) are of similar value for patients with multi-organ failure.

\section{Morbidity}

In an entirely novel disease, it is important to understand the impact on human physiology. In COVID-19, there seem to be different risks and outcomes depending on gender, with men being more affected than women, age, with elderly ( $>60$ years) more at risk than younger patients and, possibly, pregnancy putting expecting women at risk. In addition, ethnicity can have an impact on outcomes, with black, Asian and other minorities being at higher risk in the UK and the US. However, factual assessments of risks and symptoms can only be based on evidence and, thus, testing of infected patients and the general public in pandemic times is important to identify accurate risk ratios and stratify cohorts into susceptible subjects based on confirmed cases in a new disease (24).

\section{Mortality}

Mortality is defined as the number of patients who die due to a condition divided by all people who are affected by the same disease. In the COVID-19 outbreak mortality differed widely across regions (3). This was likely due to many different factors: varying stages of preparedness for the pandemic, the regional capacity of hospital and critical beds, the speed of crossinfections $\left(\mathrm{R}_{0}\right)$, the different approaches that governments took towards testing, and, partially, due to logistical organizational decisions taken. However, it is only by the means of testing that mortality rates can be accurately reported:

(I) Patients in the community may not be identified and a selected cohort of patients presenting to hospital will falsely imply a high mortality. Mortality will be overestimated in this scenario;

(II) On the other hand, deaths in the community may not be assigned to the pandemic if they are not tested. This could underestimate the true mortality rate;

(III) Both of the above scenarios may be possible at the same time, in which case an accurate mortality rate in the country/region is impossible to predict, unless the caseload is identified by widespread testing.

Without understanding these three scenarios it is difficult to judge whether a pandemic response has been successful in any given healthcare system.

\section{Public health interventions}

Due to the novelty of the virus, there were no initial antiviral treatments in the COVID-19 pandemic, and vaccination was unavailable. The main focus in the period of exponential growth of cases across the world relied therefore on public health measures that were summarized as 'non-vaccination' measures in public health, including isolation, quarantine, case identification/contact tracing, restrictions to movements and travel, as well as lockdowns. Although these measures do not cure any infected subject, they provide essential time for the healthcare system to cope with the number of patients and spread out the caseload over a longer period. The risk is that without such actions an exponential increase in the number of patients quickly leads to a saturation of existing healthcare facilities, even in the best funded systems $(3,6)$. Saturation of critical care beds would have a devastating effect on the mortality during a pandemic, and frontline staff would have the challenging task of triaging patients who arrive at the hospital when there is no capacity available.

The caseload is influenced by the regulations and public restrictions that are put in place by national governments (25), ideally in accord with international guidelines (26). A lockdown, however, has knock-on effects on physical and mental health and may be more acceptable to some populations, as previously experienced during influenza pandemics (27). Furthermore, there may be an indirect impact, a displacement effect, on patients that do not have COVID-19 infections, but who are unable to seek attention for their health concerns due to full hospitals, lack of ambulances and shut down services. Most healthcare systems have regularly updated statistics on morbidity and mortality across different regions and hospitals (28). Following normalization of any pandemic it is essential to compare routine morbidity and mortality numbers, as well as Accidents and Emergencies (A\&E) numbers to the ones registered during the pandemic. This will elucidate any indirect impact of the pandemic on non-infected patients, and there were early reports in the UK that $\mathrm{A} \& \mathrm{E}$ attendance was down by $50 \%$ during the pandemic when compared to the previous year.

Lastly, it greatly matters whether, and to what extent, government and society supports people in lockdown, with social and monetary means (29), as well as promoting mental health and physical exercise, and providing advice and information programmes (15). 


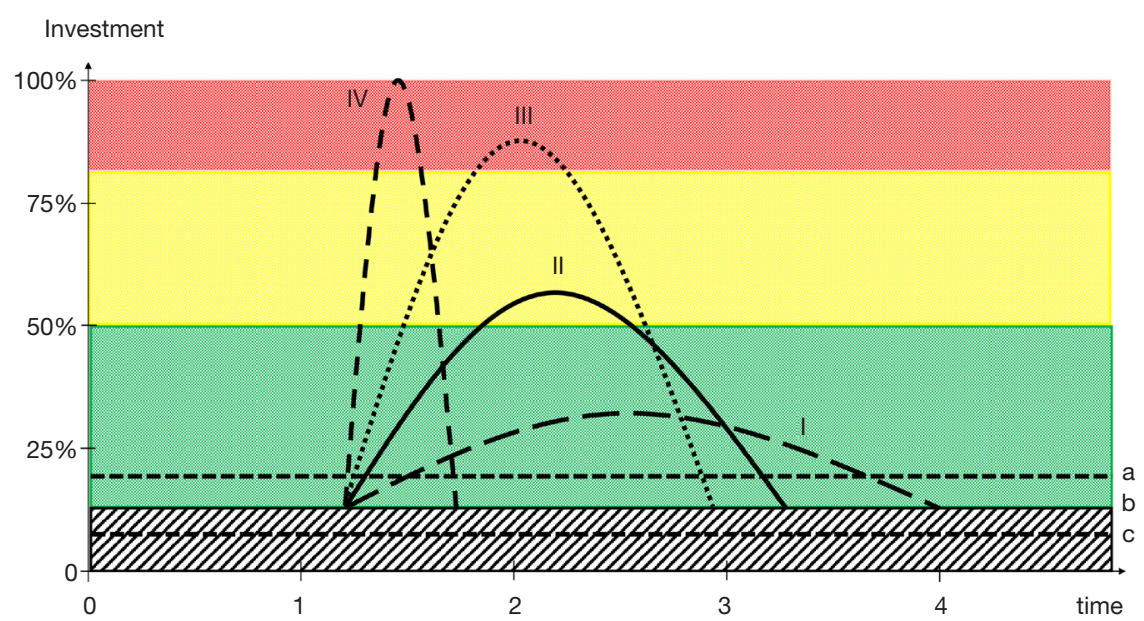

Figure 2 Schematic illustration of healthcare investment required for a pandemic response. Most healthcare systems invest a set percentage of their Gross Domestic Product (GDP) into healthcare (average indicated by the black striped box, b, with a and c indicating higher or lower investments, respectively). Shortly after ' 1 ', investment for a pandemic response is required as indicated by curves I-IV: (I) low levels of additional investment, largely covered by higher baseline investment, as defined by \% of GDP, are possible for national budgets to sustain for longer. (II) Most countries will require a response similar to this model, significant investment above baseline for a relevant duration, impacting on other economic factors, as indicated by the yellow box. (III) Several countries will have assigned a sudden surge in investment, significantly impacting on most other resources during the pandemic. (IV) This is a 'wartime-like' scenario in assigning all available resources to the pandemic resolution; the entire economy is affected (red box). However, it is likely that this level of investment cannot endure prolonged periods, as the regular economy comes to a standstill. Time in arbitrary units, investment in estimated \% of GDP. Early investment might allow for savings later on, as a full outbreak of a pandemic might be averted.

\section{Hospital occupancy rate}

High value healthcare aims to reach the entire population with the resources available. In an ideal world, any treatment would be available to everyone. However, even in the most advanced healthcare systems this remains a long-term goal. It is therefore essential to find the right balance between healthcare demand and available resources, a process that involves different parties: (I) the people; (II) healthcare delivery services, that may also act as advocates on behalf of the patients; and (III) funding providers, who can be governments, insurers, or private providers.

Pandemic resource allocation can lead to expansion of existing healthcare structures, but only by limited margins, e.g. being limited by the national GDP or possible debt. The rate at which expansion is possible and the extent to which this can happen define whether the sudden clinical case load imposed on the system can be compensated or not. The responsiveness to expand existing infrastructure by a defined margin is an important factor to cope with the peak during a pandemic. Well invested systems may have an advantage, as they require less expansion from a higher baseline; the difference between baseline and peak investment (delta) is also smaller than in under-invested structures and it is likely that adjustments can be made faster. Lastly, expansion impacts on non-healthcare related resources to a lesser degree if previous investment was higher (Figure 2).

It is important to consider the average turnover rate of cases in a pandemic. Hospital bed occupancy rate, typically recommended to be about $80-85 \%$ in non-pandemic times $(9,10)$, needs to be re-adjusted and targets will be beyond the regular $100 \%$ bed capacity of non-pandemic times. The ability to recruit additional bed capacity, particularly critical care beds, in proportion to the turnover rate can be defined as the pandemic mobilisation index. A higher index indicates a more flexible healthcare system and makes it more likely that the system will cope with the demand ("compensated"); a low index indicates that it is more likely the system will not be sufficiently prepared and may be at risk of saturation (“decompensated”; Figure 1).

Typically, adjustments to healthcare infrastructure require a cost-benefit analysis, for example by calculating the cost-effectiveness (QALY), but this may not be possible during a sudden pandemic and raises questions as to whether decisions taken during the pandemic can be 
scrutinized under general principles.

\section{Healthcare structure}

The structure of the healthcare system prior to any pandemic influences its flexibility to generate a response. Social and population-based systems covering the entire population will have certain advantages, and will have systems whereby workforces and resources can be exchanged quickly following the solidarity principle. Rivalry in fragmented healthcare systems may be disadvantageous for the provision of sufficient flexibility when there is a sudden demand. However, governments have emergency powers in a pandemic scenario and can use them, wisely and early, in an emerging pandemic scenario to create a concerted and optimal response (25).

\section{Discussion}

Understanding the direct and indirect effects of the COVID-19 pandemic is important when preparing for future pandemics. Decisions that lead to delays early on in a pandemic may prove very costly later on. A comparative analysis of different healthcare systems can identify the factors related to load and capacity of existing healthcare structures that need to be addressed in non-pandemic times. Currently, many aspects of the crisis management of the COVID-19 pandemic remain contentious across the world. The COVID-19 pandemic is arguably the most demanding non-wartime scenario in living memory that people across the globe have had to endure. This is, however, of little comfort to policymakers who face growing public unease during this unprecedented period. The expectation will be that in the future all countries are better prepared for pandemics.

\section{Comparison to non-pandemic mortality}

Whilst every death in the COVID-19 pandemic is a tragic story, it is important to compare this pandemic with nonpandemic times. How does current mortality compare to non-pandemic times? In New York (NY) State, one of the most affected regions in the world (3), septicaemia ranked $9^{\text {th }}-10^{\text {th }}$ amongst leading causes of death in the years 2014-2017, with 2,296-2,601 deaths per annum. Deaths in NY caused by non-communicable disease lead the tables in non-pandemic times; heart disease with 45,000 deaths per annum and cancer with 35,000 . The ten most common conditions that led to death caused 113,651-117,466 annual deaths between 2014-2017 (NY), an average rate of almost
10,000 deaths each month for NY only (30). The question will be in a year's time to compare these data with the number of excess deaths in New York during the pandemic.

\section{Lessons to learn}

\section{Investment}

A well-invested healthcare system is likely to cope better and faster with a pandemic. This includes, but is by no means limited to, hospital beds, critical care capacity and sufficient PPE and ventilators. It also requires a long-term strategy beyond today, to invest in research and development, which play an important role to prepare for unforeseen scenarios, and can help to provide testing capacity and new drugs, or help with tracing and PPE. Currently, spending on healthcare differs remarkably, even between highincome countries, and future policymakers should consider whether the healthcare budget is appropriate to allow for extraordinary circumstances.

Laboratories that are well funded and undertaking clinical trials are more likely to respond quickly to requests for testing, development of new vaccinations, and provision of new drugs. Equipment, particularly critical care equipment, including ventilators, ECMO and renal replacement therapy, needs to be available in sufficient quantity when a pandemic strikes, as delays can mean that lives are lost.

\section{Medical and nursing staff}

The number of doctors and nurses per 1,000 population differs by about a factor of two in high-income countries (8). The training and development of medical frontline staff in times of a pandemic requires advanced planning. It may be appropriate to train large numbers of frontline healthcare staff, particularly nurses and doctors, in annual mandatory sessions, covering critical care beyond conventional basic/ advanced life support (e.g., equipment/ventilators/PPE).

\section{Integrated health and care services}

Intelligent and novel pathways and standard operating procedures (SOP) that have been developed during the COVID-19 pandemic, including surge work rotas, stepwise opening of new units and sharing of devices to serve more cases, need to be put in place to prepare for similar events in future. Planning for survivors and preparing for capacity during the stepdown procedures, including rehabilitation and weaning units with sufficient expertise, and therapist support, and long-term care arrangements out of hospital are helpful for patients as they try to achieve normality. 
There are many lessons to be learned from this pandemic, across each affected service, in healthcare, care homes, and in the general population as well as by policymakers and business. Every branch of society should review their preparations and standard operating procedure for similar events and create a hierarchy of priorities, set goals for achievements (short-, midand long-term). It is our duty to ask inconvenient questions and learn from what went well during the pandemic, as well as what did not go well, once the pandemic is under control.

\section{Acknowledgments}

Professor Steier's contribution was partially supported by the National Institute for Health Research (NIHR) Biomedical Research Centre based at Guy's and St Thomas' NHS Foundation Trust and King's College London. The views expressed are those of the authors and not necessarily those of the NHS, the NIHR or the Department of Health. Funding: None.

\section{Footnote}

Conflicts of Interest: Both authors have completed the ICMJE uniform disclosure form (available at http://dx.doi. org/10.21037/jtd-2020-054). Prof. JS serves as an unpaid editorial board member of Fournal of Thoracic Disease from May 2013 to Apr 2021. JM has no conflicts of interest to declare.

Ethical Statement: The authors are accountable for all aspects of the work in ensuring that questions related to the accuracy or integrity of any part of the work are appropriately investigated and resolved.

Open Access Statement: This is an Open Access article distributed in accordance with the Creative Commons Attribution-NonCommercial-NoDerivs 4.0 International License (CC BY-NC-ND 4.0), which permits the noncommercial replication and distribution of the article with the strict proviso that no changes or edits are made and the original work is properly cited (including links to both the formal publication through the relevant DOI and the license). See: https://creativecommons.org/licenses/by-nc-nd/4.0/.

\section{References}

1. Kluge HHP on behalf of the World Health Organization. WHO announces COVID-19 outbreak a pandemic.
Available online: http://www.euro.who.int/en/healthtopics/health-emergencies/coronavirus-covid-19/news/ news/2020/3/who-announces-covid-19-outbreak-apandemic, accessed 31-03-2020.

2. World Economic Forum. It could take three years for the US economy to recover from COVID-19. Available online: https://www.weforum.org/agenda/2020/03/ economic-impact-covid-19/, accessed 31-03-2020.

3. Johns Hopkins Coronavirus Resource Centre. Coronavirus Covid-19 Global Cases by the Center for Systems Science and Engineering. Available online: https://coronavirus.jhu. edu/map.html, accessed 31-03-2020.

4. UK Government. Coronavirus-19: what you need to do. Available online: https://www.gov.uk/coronavirus, accessed 31-03-2020

5. BBC News. Coronavirus: the world in lockdown in maps and charts. Available online: https://www.bbc.co.uk/news/ world-52103747, accessed 11-04-2020.

6. Ferguson NM, Laydon D, Nedjati-Gilani G, et al. Report 9: Impact of non-pharmaceutical interventions (NPIs) to reduce COVID-19 mortality and healthcare demand. 1603-2020. Available online: https://www.imperial.ac.uk/ mrc-global-infectious-disease-analysis/covid-19/report-9impact-of-npis-on-covid-19/, accessed 10-04-2020.

7. Hobbs FDR, Bankhead C, Mukhtar T, et al. Clinical workload in UK primary care: a retrospective analysis of 100 million consultations in England, 2007-14. Lancet 2016;387:2323-30.

8. Papanicolas I, Woskie LR, Jha AK. Health Care Spending in the United States and Other High-Income Countries [published correction appears in JAMA. 2018 May 1;319(17):1824]. JAMA 2018;319:1024-39.

9. NHS England. Available online: https://www.england. nhs.uk/wp-content/uploads/2019/05/Adult-Critical-CareService-Specification-FINAL.pdf, accessed 04-04-2020.

10. Valentin A, Ferdinande P; ESICM Working Group on Quality Improvement. Recommendations on basic requirements for intensive care units: structural and organizational aspects. Intensive Care Med 2011;37:1575-87.

11. Lawrence Dunhill. Exclusive: Intensive care staffing ratios dramatically diluted. HSJ 2020; Available online: https:// www.hsj.co.uk/exclusive-intensive-care-staffing-ratiosdramatically-diluted/7027214.article, accessed 08-04-2020.

12. NHS England. Available online: https://www.england.nhs. $\mathrm{uk} /$ participation/get-involved/volunteering/nhs-volunteerresponders/, accessed 14-04-2020.

13. Wikipedia. List of countries by hospital beds. Available 
online: https://en.wikipedia.org/wiki/List_of_countries_ by_hospital_beds, accessed 11-04-2020.

14. National Institute for Health and Care Excellence (NICE). NICE guideline 94: Chapter 39, Bed Occupancy. NICE 2018; ISBN 978-1-4731-2741-8.

15. National Institute for Health and Care Excellence. Covid-19 rapid guideline: critical care in adults. Available online: https://www.nice.org.uk/guidance/ ng159/chapter/2-Admission-to-critical-care, accessed 17-04-2020.

16. British Sleep Society. BSS statement on sleep-related advice during the coronavirus (COVID-19) pandemic. Available online: https://www.sleepsociety.org.uk/wpcontent/uploads/2020/04/BSS-statement-on-sleeprelated-advice-during-the-coronavirus-Covid-19pandemi.pdf, accessed 17-04-2020.

17. Ranney ML, Griffeth V, Jha AK. Critical Supply Shortages - The Need for Ventilators and Personal Protective Equipment during the Covid-19 Pandemic. N Engl J Med 2020;382:e41.

18. Royal College of Physicians, London. COVID-19 and its impact on the NHS workforce. Available online: https:// www.rcplondon.ac.uk/news/covid-19-and-its-impact-nhsworkforce, accessed 14-04-2020.

19. Hasell J, Ortiz-Ospina E, Mathieu E, et al. Coronavirus Disease (COVID-19) - Statistics and Research, the Our World in Data Covid-10 Testing dataset. Our World in Data 31-03-2020; Available online: https://ourworldindata. org/covid-testing, accessed 09-04-2020.

20. Connolly B, Douiri A, Steier J, et al. A UK survey of rehabilitation following critical illness: implementation of NICE Clinical Guidance 83 (CG83) following hospital discharge. BMJ Open 2014;4:e004963.

21. Phillips C. What is a QALY? In "What is...?" Series, 2nd edition, 2009. Hayward Medical Communications, Swansea. Available online: http://www.bandolier.org.uk/

Cite this article as: Steier J, Moxham J. The load and capacity model of healthcare delivery: considerations for the crisis management of the COVID-19 pandemic. J Thorac Dis 2020;12(6):3022-3030. doi: 10.21037/jtd-2020-054
painres/download/whatis/QALY.pdf, accessed 14-04-2020.

22. Sanche S, Lin YT, Xu C, et al. High Contagiousness and Rapid Spread of Severe Acute Respiratory Syndrome Coronavirus 2. Emerg Infect Dis 2020. doi: 10.3201/ eid2607.200282.

23. Zhu N, Zhang D, Wang W, et al. A Novel Coronavirus from Patients with Pneumonia in China, 2019. N Engl J Med 2020;382:727-33.

24. Guan WJ, Ni ZY, Hu Y, et al. Clinical Characteristics of Coronavirus Disease 2019 in China. N Engl J Med 2020;382:1708-20.

25. UK Government. Emergency bill to strengthen coronavirus (COVID-19) response plans. Available online: https://www.gov.uk/government/news/emergency-bill-tostrengthen-coronavirus-covid-19-response-plans, accessed 16-04-2020.

26. World Health Organization. COVID 19 Strategy update 14 April 2020. Available online: https://www.who.int/ publications-detail/strategic-preparedness-and-responseplan-for-the-new-coronavirus, accessed 16-04-2020.

27. SteelFisher GK, Blendon RJ, Ward JR, et al. Public response to the 2009 influenza A H1N1 pandemic: a polling study in five countries. Lancet Infect Dis 2012;12:845-50.

28. Public Health England. NHS Atlas of Variation. Available online: http://tools.england.nhs.uk/images/ RespiratoryAtlas/atlas.html, accessed 16-04-2020.

29. UK Government. Claim for Wages through the Coronavirus Job Retention Scheme. Available online: https://www.gov.uk/guidance/claim-for-wages-through-thecoronavirus-job-retention-scheme, accessed 21-04-2020.

30. Center for Disease Control and Prevention (CDC) / Centre for National Statistics. Stats for the State of New York 201417. Available online: https://www.cdc.gov/nchs/pressroom/ states/newyork/newyork.htm, accessed 14-04-2020. 